\title{
Determination of the Ice Load Acting on the Up-bending and Down-bending Cone of the Marine Structure Pile
}

\author{
Lingqiang Yang 1, a, Rui Gao ${ }^{1, b}$, Yan Wang ${ }^{1, c}$ \\ ${ }^{1}$ School of Civil engineer and architecture, University of Jinan, Jinan, Shandong province, China \\ acea_yanglq @ujn.edu.cn, cea_Gaor @ujn.edu.cn b, eo_wangy @ujn.edu.cn c
}

\begin{abstract}
Keywords: continuous and non-continuous body; the unit force method of non-coordinate analysis; imaginary movable loading system ice load cones; ice load.

Abstract. Ice load is very important for the design of marine structures at low temperature areas. In order to reduce the ice load to the marine structure, it is common to pile with up-bending and down-bending cones. Therefore, the determination of the ice load acting on the up-bending and down-bending cone is crucial for the structure design. In this paper, unit force method was used to determine the ice load based on theory of structure mechanics and non-coordinate analysis of non-continuous body. Since the contact pressure between the cone and ice is unknown, a movable loading system with virtual stiff components is proposed and used to study the ice load on the up-bending and down-bending cone with multivariate nonlinear regression method. The result shows that with the up-bending and down-bending cone, the ice load acting on the pile is largely reduced.
\end{abstract}

\section{Introduction}

For the design of marine structures, the load of the sea ice on the structure is very important. In fact, it is a contact load, changes with the ice-breaking process, between the flowing sea ice and the structure. From the standpoint of engineering design for marine structure, the maximum value of the load is important to determine and first of all, the sea ice stiffness need to be investigated. For many marine structures, the piles are in direct contact with the sea ice and the geometry of the pile is an importance factor influences the ice load. Using the up-bending and down-bending cone, the ice-load to the structure could be significantly reduced. Up-bending and down-bending cones have been widely applied in marine structure construction at Bo-hai Ocean in China. As a result, the method of piling with up-bending and down-bending cones is becoming more and more popular in order to increase the structure safety and reduce cost.

Many researcher focused on the interaction Moored structures and sea ice to ascertains the load. The ice drift speed plays a vital role in the dynamics of the ice load process for both broken ice, level ice and ridge ice, while the mooring stiffness determines some of the main properties of the vessel. The effect of ice drift speed on moored structures has been considered by Toyama and Yashima (1985), Løset et al. (1998), Comfort et al. (1999), Wright (1999), Aksnes and Bonnemaire (2009), Bonnemaire et al. (2009), among others, while mooring stiffness effects have been discussed by Jensen et al. (2008), Aksnes and Bonnemaire (2009), Bonnemaire et al. (2009). Most of these studies were based on data from full-scale experiences with the cylindrical floater Kulluk or model test data. It is different to pile with up-bending and down-bending cone in marine structures. In addition to the dramatic complexity of the broken process of sea ice, it is very necessary to study the load acting on the pile with up-bending and down-bending cone systematically. Some researchers studied the ice load on the cone. Schwarz(1982) proposed the equation for the ice load as:

$$
\mathrm{P}=\alpha D^{\omega} T^{\varphi} f_{c}
$$

Where $\mathrm{P}$ is the ice load; $\mathrm{D}$ is the diameter of the pile; $\mathrm{T}$ is the temperature; $f_{c}$ is the ice compressive strength; ${ }^{\alpha},{ }^{\omega}$, and ${ }^{\varphi}$ are coefficients.

MCPRC(2010) proposed the equation for the ice load as:

$$
\mathrm{P}=\alpha \mathrm{DT} f_{c}
$$


In both equations (1) and (2), it is considered the ice load is not correlated to the tensile strength of the sea ice. However, G.W. Timco (2010) pointed out that the tensile strength is a fundamental property of sea ice. The tensile strength is a key parameter in defining the failure envelope of sea ice. It also represents a key failure mode when ice interacts with marine structure.

\section{Mechanics Model for the ice Load Analysis}

The ice load acting on the pile equals the crushing strength. Therefore, the ice breaking process is studied and the crushing strength is investigated to determine the ice load.

Assume the sea ice is an infinite panel. The ice panel has its gravity and is floated by sea water and reaches equilibrium. Considering the flow and incompressible of the sea water, there is no plane stress generated. In addition, at the boundary between the contact of the ice panel and the cone, there is contact load along the boundary and therefore generate the stress in the ice panel. Therefore, the stress analysis of the ice panel can be simplified to a stress analysis of an infinite panel with boundary loads and no gravity.

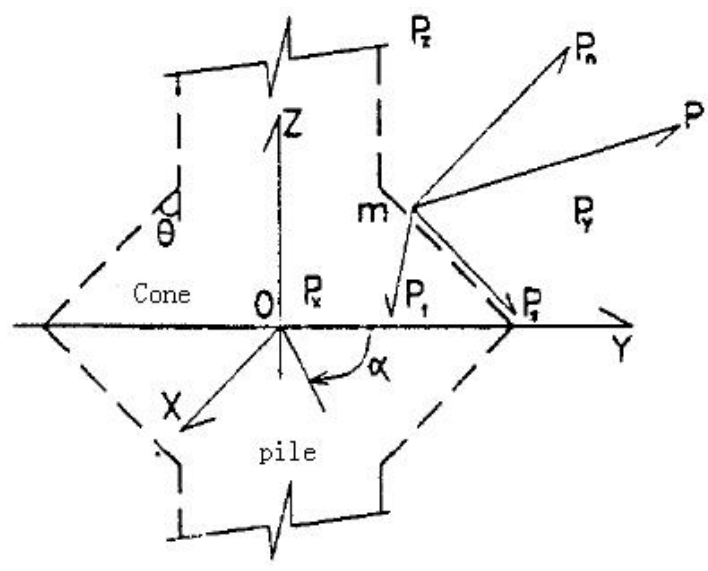

Figure 1. Contact Load between Ice Panel and Cone

Consider a random point at the contact boundary between the ice panel and the cone, three components of the contact load P are: normal Pn and two tangential Ps and Pt. Ps and Pt are the friction. At the same time, it should be noted that the ice panel is flowing and once ice panel touches the cone, the panel breaks and flow away in smaller pieces, therefore, the friction between the ice panel and the cone is negligible. The normal component of the contact load Pn is important and need to be investigated.

Component $\mathrm{Pn}$ can be decomposed again to normal component $\mathrm{Pz}$ and tangential components $\mathrm{Px}$ and Py. Pz generates the bending moment, while Px and Py generate the plane stress of the panel. The breaking process of the ice panel is the combination of the two stress states and will be studied in this paper.

In Figure 1, it shown that components $\mathrm{Pz}, \mathrm{Px}$ and $\mathrm{Py}$ are the functions of locations and therefore $\operatorname{Pz}\left({ }^{\alpha}\right), \operatorname{Px}\left({ }^{\alpha}\right), \operatorname{Py}\left({ }^{\alpha}\right)$. They are also related since they are all components of Pn. The integral of horizontal component $\mathrm{Py}\left({ }^{\alpha}\right)$ is the horizontal driving load acted on the pile PY; The integral of horizontal component $\operatorname{Px}\left({ }^{\alpha}\right)$ is the horizontal resisting load of the cone Px; The integral of the vertical component $\mathrm{Pz}\left({ }^{\alpha}\right)$ is the axial eccentric force of the pile PZ. When the ice panel hits the up-bending cone, $\mathrm{Pz}$ is the axial eccentric compression. When the ice panel hits the down-bending cone, $\mathrm{Pz}$ is the axial eccentric tension. 


$$
\left\{\begin{array}{l}
P_{y}=\int_{0}^{2 \pi} p_{y}(\alpha) d \alpha \\
P_{z}=\int_{0}^{2 \pi} p_{z}(\alpha) d \alpha \\
P_{x}=\int_{0}^{\pi \pi} p_{x}(\alpha) d \alpha \\
\int_{0}^{2 \pi} p_{x}(\alpha) d \alpha=0
\end{array}\right.
$$

At any point on the cone:

$$
P_{\mathrm{n}}=\sqrt{P_{x}^{2}+P_{y}^{2}+P_{z}^{2}}
$$

The total load of the pile:

$$
\mathrm{P}=\sqrt{P_{y}^{2}+P_{x}^{2}}
$$

The angle between the load and the pile

$$
\beta=\operatorname{tg}^{-1} \frac{P_{y}}{P_{x}}
$$

The maximum values of PZ, PY and PX are related to the stiffness of the ice panel structure. Therefore, the failure mechanism should be investigated for an ice panel with boundary loading.

\section{Assumptions}

The ice load acting on marine structures is the impact force of the flowing sea ice to the structure and it equals the crushing strength of the ice. The crushing strength of the ice is related to the geometry of the ice panel and the mechanical properties of the ice material. The ice panel has an initial curvature due to wave. The mechanical properties of the ice material includes temperature, chemical ingredients, sand content, strain velocity during the flow and wind velocity, etc. It is very difficult to include all these factors in the analysis of ice loading, therefore, the following assumptions are made in this analysis:

1. Neglect the dynamic responses of the marine structure and the ice. Neglect the elastic deformation of the pile.

2. Full contact between ice and the cone surface, neglect the friction.

3. Linear stress strain relation within the compression strength fc; pure plastic after reaching the compression strength $\mathrm{fc}$; brittle failure after reaching tensile strength. The stress strain relation of the ice material is shown in Figure 2.

\section{Apply Ice Boundary loading}

Ice boundary load is not only the function of the location, but also the function of the angle ${ }^{\theta}$ (see Figure 3). Besides, the ice boundary load is the function of displacement of the ice boundary and the degree of crushing. Therefore, the ice load function is changing during the loading process. As shown in Figure 3, the total load acting on the pile, and its components are important for the design of the pile, we can assume an upside down umbrella loading, where the stiffness of beams is infinite and hinge at point $\mathrm{B}$, a point hinges with the ice boundary, during the process of the deformation, direction angle ${ }^{\theta}$ of the beams remain unchanged but the length of the beam can be adjusted. Therefore, we can insert horizontal load Py at point B. The maximum elastic capacity P0 of the ice panel is Py at the initial failure. The maximum load bearing capacity of the ice panel is the Py when the panel starts to lose its stability. The loading distribution of the ice boundary including the normal component $\operatorname{Pz}\left({ }^{\alpha}\right)$ and two tangential component $\operatorname{Px}\left({ }^{\alpha}\right)$ and $\operatorname{Py}\left({ }^{\alpha}\right)$ can also be derived from equilibrium.

This upside-down umbrella loading is the assumed loading system of the ice boundary, it is a movable system composed of stiff components. B point is the loading point of the loading system. 


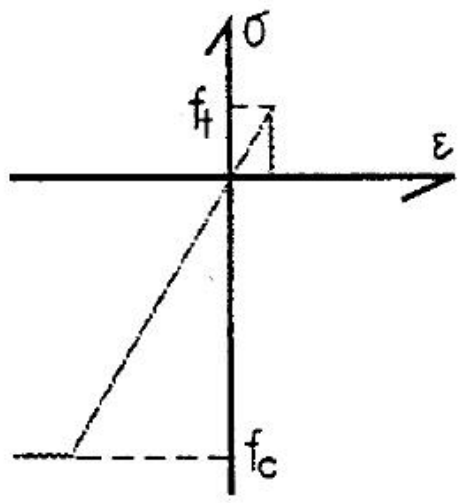

Figure 2. Stress Strain Relation of the Ice Material

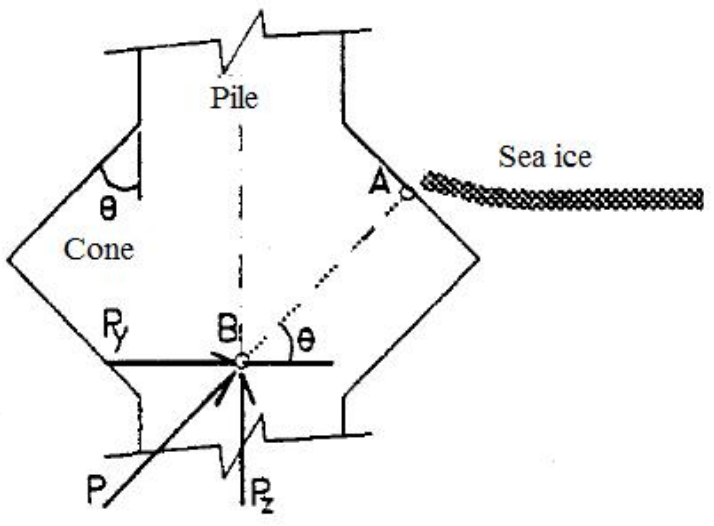

Figure 3. Section Loading

\section{Analytical Method of the Ice-breaking Process - Unit Force Method}

The ice-breaking process is a non-coordinate analysis of the continuous body to non-continuous body. A simple method is presented below considering the aforementioned assumptions.

Apply a unit force $\mathrm{p}=1$ at the loading point to the loading system of the ice boundary and calculate the stress field of the ice panel, locate the point where it has the maximum stress. Using the assumptions of the stress-strain relations, the ice load at the point can be derived and it equals the ice load at the initial failure, this load is marked as P0. The stress field is a critical field at the initial failure and it is non-stable. The stable stress field can be derived by re-evaluating the field after removing the continuity of the point. And the discontinuous ice panel is the panel that has reduced one degree of freedom. Apply unit force $p=1$ at initial failure point of the panel that has reduced one degree of freedom, find the critical point and its ice load to be used for the next iteration P1, repeat the process to get $\mathrm{p} 2, \mathrm{p} 3, \ldots, \mathrm{pn}$. When $\mathrm{Pk}+1<\mathrm{Pk}(\mathrm{k}+1<\mathrm{n})$, it means the ice panel has crushed and $\mathrm{Pk}$ is the maximum ice load, Pc. If Pc cannot be found, the ice load when the ice panel starts to lose its stability is taken as the maximum ice load Pc.

\section{Conclusions}

With the up-bending and down-bending cone, there is an axial eccentric compression or tensile load acting on the pile. When the ice panel hit the up-bending cone, the axial eccentric load is compression load which strengthens the stability of the pile, when the ice panel hit the down-bending cone, the axial eccentric load is tensile load and it reduces the stability of the pile.

\section{Acknowledgements}

This work was financially supported by the Shandong Natural Science Foundation (ZR2014EL038).

\section{References}

[1] A. Suyuthi. B.J. Leira. K. Riska. Short term extreme statistics of local ice loads on ship hulls. Cold Regions Science and Technology. 82 (2012) 130-143.

[2] A. Suyuthi, B.J. Leira, K. Riska. Statistics of local ice load peaks on ship hulls. Structural Safety. 40 (2012) 1-10. 